\title{
Complete high-precision entropic sampling
}

\author{
Ronald Dickman* and A. G. Cunha-Netto ${ }^{\dagger}$ \\ Departamento de Física, Instituto de Ciências Exatas, \\ and National Institute of Science and Technology for Complex Systems, \\ Universidade Federal de Minas Gerais, C.P. 702, \\ 30123-970 Belo Horizonte, Minas Gerais, Brazil
}

\begin{abstract}
Monte Carlo simulations using entropic sampling to estimate the number of configurations of a given energy are a valuable alternative to traditional methods. We introduce tomographic entropic sampling, a scheme which uses multiple studies, starting from different regions of configuration space, to yield precise estimates of the number of configurations over the full range of energies, without dividing the latter into subsets or windows. Applied to the Ising model on the square lattice, the method yields the critical temperature to an accuracy of about $0.01 \%$, and critical exponents to $1 \%$ or better. Predictions for systems sizes $L=10-160$, for the temperature of the specific heat maximum, and of the specific heat at the critical temperature, are in very close agreement with exact results. For the Ising model on the simple cubic lattice the critical temperature is given to within $0.003 \%$ of the best available estimate; the exponent ratios $\beta / \nu$ and $\gamma / \nu$ are given to within about $0.4 \%$ and $1 \%$, respectively, of the literature values. In both two and three dimensions, results for the antiferromagnetic critical point are fully consistent with those of the ferromagnetic transition. Application to the lattice gas with nearest-neighbor exclusion on the square lattice again yields the critical chemical potential and exponent ratios $\beta / \nu$ and $\gamma / \nu$ to good precision.

PACS numbers:
\end{abstract}

Keywords: Monte Carlo simulation, entropic sampling, Ising model, lattice gas

* e-mail address: dickman@fisica.ufmg.br

$\dagger$ e-mail address: agcnetto@fisica.ufmg.br 


\section{INTRODUCTION}

A simple and reliable Monte Carlo method, capable of estimating thermodynamic properties at any desired temperature using a single simulation, has been sought for many years [1-4]. Such a method could in principle offer a great advantage over Metropolis Monte Carlo and cluster algorithms, which require a separate study for each temperature of interest. The information required to study the full temperature range is contained in the number of configurations $\breve{\Omega}(E, L)$ as a function of energy $E$, and associated microcanonical averages [for example, the mean magnetization $\langle m\rangle(E, L)$ ], on a lattice of $L^{d}$ sites. Wang-Landau sampling (WLS) [5, 6] is currently the most widely used such entropic sampling method. Various improvements to the basic WLS scheme have been suggested [7-10]. In WLS of larger systems the range of energies (or of other relevant quantities) is divided into subsets and each such "window" is sampled separately. The latter procedure has been found to distort the estimates for $\breve{\Omega}(E, L)$ in some cases [8, 10].

Despite the broad interest in WLS, relatively few works have been published in which critical exponents are determined using the method. Important exceptions to this general trend are the studies of Malakis and coworkers [11 15], who use a modified WLS procedure to find the critical exponents of several pure and disordered spin models. Their method involves a two-stage process, first using WLS with the energy range divided into windows to identify the critical energy subspace (i.e., the range of energies needed to sample the critical region adequately), followed by WLS restricted to the critical range. In Ref. [22] the present authors obtained fair results for the critical exponents of the lattice gas with nearest-neighbor exclusion, using WLS with adaptive windows.

Here we describe a high-precision entropic simulation method that samples the full configuration space without using windows. Two fundamental aspects of the method are: (1)

successive refinement of an initial estimate for the $\breve{\Omega}(E, L)$ on the basis of simulations starting from a diverse set of initial configurations, and (2) use of the final result at size $L$ to generate a good preliminary estimate for $\breve{\Omega}\left(E, L^{\prime}\right)$ for size $L^{\prime}>L$. After describing the method in Sec. II, we present, in Sec. III, applications to the Ising model on the square and simple cubic lattices, and to the lattice gas with nearest-neighbor exclusion. A summary and conclusions are given in Sec. IV. 


\section{METHOD}

Consider a statistical model with a discrete configuration space, and let $\vartheta$ denote a variable (or set of variables) characterizing each configuration, such as energy or particle number. For a given system size, knowledge of the number $\breve{\Omega}(\vartheta)$ of configurations, for all allowed values of $\vartheta$, permits one to evaluate the partition function and associated thermal averages for any desired temperature. Entropic sampling methods use Monte Carlo simulation to furnish estimates of the configuration numbers, which we denote by $\Omega(\vartheta)$, reserving $\breve{\Omega}(\vartheta)$ to denote the exact values, which are in general unknown. The basic idea of entropic sampling is as follows. Let $E(\mathcal{C})$ denote the energy of configuration $\mathcal{C}$. Consider a simulation method that generates configurations from the probability distribution

$$
P(\mathcal{C}) \propto \frac{1}{\breve{\Omega}[E(\mathcal{C})]},
$$

i.e., the probability of configuration $\mathcal{C}$ is inversely proportional to the number of configurations having the same energy. This could be done using an acceptance probability

$$
p\left(\mathcal{C}^{\prime}\right)=\min \left[\frac{\breve{\Omega}(E)}{\breve{\Omega}\left(E^{\prime}\right)}, 1\right] .
$$

(As usual, when the new configuration $\mathcal{C}^{\prime}$ is rejected, the current configuration $\mathcal{C}$ is counted again in the sample.) Let $H(E)$ be the number of times energy $E$ occurs in the sample. In a simulation that follows Eq. (11),$\langle H(E)\rangle=$ Const., independent of $E$. Of course, the $\breve{\Omega}(E)$ are in general unknown. If we run the simulation using a set of estimated values, $\Omega(E)$, in place of $\breve{\Omega}(E)$ in Eq. (11), then the better the estimate, the more uniform the histogram $H(E)$ should be. The expected number of visits to energy $E$ when we use an estimate $\Omega(E)$ to define $p_{a}(\mathcal{C})$ is

$$
\langle H(E)\rangle \propto \frac{\breve{\Omega}(E)}{\Omega(E)}
$$

Suppose we begin using a guess, $\Omega_{0}(E)$, for $\breve{\Omega}(E)$. Simulating for a sufficiently long time, so that $H(E) \approx\langle H(E)\rangle$ for all energies $E$, we may refine our guess, using Eq. (3) to write

$$
\Omega_{1}(E)=\frac{H_{1}(E)}{\bar{H}_{1}} \Omega_{0}(E)
$$


where $H_{1}(E)$ is the number of times energy $E$ occurs in the simulation, and $\bar{H}_{1}$ is the average of $H_{1}(E)$ over all allowed energies. Equation (4) is the basis of our simulation method. The idea is to iterate this procedure, starting from an initial guess, enough times so that the final approximation, $\Omega_{N}$, is very close to the true distribution. (During the final iteration, the microcanonical averages required to calculate thermal averages are also determined.)

For the method to yield a reliable estimate for $\breve{\Omega}(E)$, it is essential that the space of configurations be sampled long enough, and broadly enough, that at each step $j=1, \ldots, N$, the observed histogram $H_{j}(E)$ is close to its expected value, that is, $H_{j}(E) \propto \breve{\Omega}(E) / \Omega_{j-1}(E)$, for all allowed values of the energy. If this condition is satisfied at each iteration $j$, we expect the estimates $\Omega_{j-1}(E)$ to approach the exact values.

The preceding discussion suggests that, to converge, the configuration space must be sampled well at each iteration. Our numerical experiments confirm that better results are obtained using a relatively small number of iterations, with a relatively large number of lattice updates (LUDs) [16] than with a larger number of iterations, each having a smaller number of updates, as shown in the following section. In the studies reported below, for example, we use $N=5$ steps, each consisting of a set of ten simulations, each of which runs for $N_{U}=10^{7}$ LUDs.

To ensure adequate sampling of the full range of energies, the simulations in a given iteration use a variety of initial configurations. For the Ising model on the square and simple cubic lattices, for which the number of configurations is invariant under (1) inversion of all spins, and (2) inversion of all spins on one sublattice, we use the following set of initial conditions: (1 and 2) random spin orientations; (3) all spins up; (4) all spins down; (5) all but two neighboring spins up; (6) all but two neighboring spins down; (7 and 8) all spins on one sublattice up, the rest down; (9 and 10) configurations (7) or (8) with a pair of neighboring spins inverted. Thus there are four low-energy initial configurations, four of high energy (but of low energy for the antiferromagnetic model), and two that have $E \approx 0$. Taking a certain liberty with terminology, we call this approach tomographic sampling of the distribution $\Omega_{j}$, since studies using different starting points are pooled at each iteration.

A key question concerns the initial estimate, $\Omega_{0}$, for the number of configurations. Here it is important to note that, in terms of the energy density $e=E / L^{d}$, the microcanonical entropy density, 


$$
s(e, L) \equiv \frac{1}{L^{d}} \ln \breve{\Omega}\left(L^{d} e, L\right)
$$

depends on system size $L$ rather weakly. Thus the final result for $s(e, L)$ serves as a rather good first approximation for a larger system size, $L^{\prime}$. We use a simple initial guess (for example, $\Omega(E)$ uniform over the set of allowed energies, or a mean-field approximation) for some small initial system size. For small $L$ the simulation rapidly converges to a good estimate of $\breve{\Omega}(E, L)$, and the resulting entropy density $s(e, L)$ is then used to generate the initial guess $\Omega_{0}\left(E, L^{\prime}\right)$ for the next system size in the sequence.

\section{A. Implementation}

All the studies reported here use periodic boundaries; other boundary conditions are readily implemented. The Ising spin configurations are classed by the bond number $n$, i.e., the number of nearest-neighbor pairs of spins having the same orientation. On the square lattice the allowed values are $n=0,4,6, \ldots, n_{\max }-4, n_{\max }$ with $n_{\max }=2 L^{2}$. (The energy is $E=2\left(L^{2}-n\right)$.) To begin, we set the initial distribution $\Omega_{0}(n)$. Due to the extremely large values these numbers may take (already $\sim \mathcal{O}\left(10^{30}\right)$ for $L=10$ ), they are represented in the form of their natural logarithm. Subsequently, the simulation procedure is called $N$ times. (In most of the studies reported here, $N=5$.) The simulation uses a single-spin-flip dynamics. The acceptance probabilities $\Omega_{j}(n) / \Omega_{j}\left(n^{\prime}\right)$ (for a transition from energy $n$ to $n^{\prime}$ ), are stored in a look-up table. (The allowed values of $\Delta n$ are $0, \pm 2$, and \pm 4 .) In addition to storing the orientation of each spin, we store the number of neighbors having the same spin orientation, as this facilitates evaluation of $\Delta n$.

Each time the simulation subroutine is called, the histogram $H_{j}(n)$ is set to zero. The latter then accumulates the number of visits to each class $n$ over $N_{\text {sim }}=10$ simulations (with varied initial configurations), each consisting of $N_{U}$ LUDs. (In principle, these studies could be carried out in parallel, on separate processors.) Once all $N_{\text {sim }}$ studies are done we update $\Omega(n)$ as per Eq. (4). The acceptance probabilities are recalculated using the new estimate, and we proceed to the next iteration, for a total of $N$ iterations. Each time a configuration $\mathcal{C}$ is generated, be it a new one (following an accepted spin flip) or a repetition (if the new configuration is rejected), we update the running sums used to calculate the microcanonical averages of the absolute magnetization $|M|$, as well as $M^{2}$ and $M^{4}$. The resulting averages 
are saved, along with $\ln \Omega_{j}(n)$, at the end of each iteration. (Note however that only the results from the final iteration, $j=N$, are used to calculate the thermodynamic properties reported below.)

As mentioned above, the initial distribution $\Omega_{0}(n, L)$ is generated on the basis of the final estimate for a smaller system size. For the smallest size $(L=10$ in the case of the square lattice) we use a simple approximation for the entropy density,

$$
s(\rho) \approx-\ln 2(\rho-1)^{2}
$$

where $\rho=n / L^{2}$. This is motivated by the fact that $\breve{\Omega}\left(n=L^{2}\right) \sim 2^{L^{d}}$ whereas $\breve{\Omega}\left(n=2 L^{d}\right)=$ $\breve{\Omega}(n=0)=2$, and that $s(\rho)$ is symmetric about $\rho=1$. (For convenience we subtract $\ln 2$ from the original definition, $s=[\ln \breve{\Omega}(n)] / L^{2}$.) For $L=10$, the distribution after the first iteration, $\Omega_{1}(n)$, is already very close to the final one, showing rapid convergence.

The quantity $h(\rho) \equiv[H(\rho)-\bar{H}] / \bar{H}$ characterizes the relative deviation of the histogram from a uniform distribution; its evolution at successive iterations is illustrated in Fig. 1. The result of the first iteration (starting from the parabolic initial approximation) shows relatively large variations, while the subsequent histograms are all flat on the scale of this figure. The detailed evolution in the subsequent iterations is shown in the inset; the final histogram is flat to about $99.5 \%$.

FIG. 1: (Color online) Ising model, square lattice: relative deviation of histogram, $h(\rho) \equiv[H(\rho)-$ $\bar{H}] / \bar{H}$, versus bond density $\rho$ for $L=10$. The black curve is the result of the first iteration, while the horizontal line corresponds to the four subsequent iterations. Inset: Detail of iterations $2-5$.

For the passage from one system size $(L)$, to the next $\left(L^{\prime}\right)$, we require an algebraic approximation for the entropy density $s(\rho, L)$. Given that $\breve{\Omega}(n)=\breve{\Omega}\left(n_{\max }-n\right)$, we first symmetrize the result about $\rho=1$, and average over pairs of neighboring values to suppress small systematic oscillations in $s$. (The latter are visible in Fig. 2, near $\rho=0$ and $\rho=2$.) This defines the symmetrized and (slightly) smoothed entropy density,

$$
\tilde{s}\left(\rho^{\prime}\right) \equiv \frac{1}{4}\left[s\left(n / L^{2}\right)+s\left((n-1) / L^{2}\right)+s\left(2-n / L^{2}\right)+s\left(2-(n-1) / L^{2}\right)\right]
$$

where $\rho^{\prime}=(2 n-1) /\left(2 L^{2}\right)$. For smaller sizes, $\tilde{s}$ is well approximated by a polynomial fit in even powers of $x \equiv \rho-1$. For larger systems, however, maintaining a good fit requires 
increasing the order of the polynomial. The fit tends to be worst near $x= \pm 1$. (In fact, one may anticipate a weak singularity, $s \sim|1-x| \ln |1-x|$ at these limits.) We find that, given the symmetry $s(-x)=s(x)$, a more convenient alternative is a Fourier cosine series:

$$
s(x)-s(1)=\sum_{j=0}^{J} a_{j} \cos \left[\frac{(2 j+1) \pi x}{2}\right] \equiv f(x)
$$

The expansion coefficients are readily calculated using the discrete approximation to the standard integral expression; the quality of the fit varies considerably with the number of terms in the series. We calculate the maximum absolute deviation $\delta \equiv \max _{x}|s(x)-f(x)|$ as a function of the number of terms, and use the value that minimizes $\delta$. For example, for $L=120$ (square lattice), we obtain the smallest $\delta\left(2.7 \times 10^{-5}\right)$ for a series with $J=9151$. We use the Fourier series for all studies of the two- and three-dimensional Ising models reported below.

The execution time (for a given number of iterations, initial configurations, and lattice updates) scales as the number of lattice sites. The largest studies reported here (for system size $L=160$ ) required approximately one week on a processor with a speed of $2.8 \mathrm{GHz}$.

\section{RESULTS}

\section{A. Ising model on the square lattice}

We apply tomographic sampling to the Ising model on the square lattice, for system sizes $L=10,20,40,80,120$, and 160. The procedure is as outlined above, except that for the two largest sizes, following the five studies using $N_{U}=10^{7}$ lattice updates, a sixth study using $N_{U}=2 \times 10^{7}$ is performed. In all cases, the results are calculated on the basis of the final study. Means and uncertainties are calculated using five or in some cases six independent simulations.

The number of configurations $\Omega(\rho)$ takes its maximum at $\rho=1$ and is (except for fluctuations) symmetric about this point. In Fig. 2 we plot the microcanonical entropy density

$$
s(\rho)=\frac{1}{L^{2}} \ln \left(\frac{\Omega(\rho)}{\Omega(1)}\right)
$$


for several system sizes. At this scale, finite-size effects are only evident near the limiting bond densities.

FIG. 2: (Color online) Microcanonical entropy density $s(\rho)$ versus $\rho$ for system sizes $L=10,20$, 40, 80, and 160 (upper to lower). The inset is a detailed view near $\rho=2$.

To perform a quantitative test of the method we compare results for the specific heat, magnetization, susceptibility, and reduced fourth cumulant with known values and with finitesize scaling theory [17, 18]. We define the specific heat per site as $c=\operatorname{var}(E) /\left(L^{d} k_{B} T^{2}\right)$, where $E$ is the total energy. (From here on we employ units such that Boltzmann's constant $k_{B}=1$.) Figure 3 shows $c(T)$ (for $L=80$ ) as obtained in five independent simulations. The curves are indistinguishable on the scale of the main graph, but a detail of the critical region does reveal some scatter in the specific heat maxima and the temperatures at which they occur. From the analysis of Ferdinand and Fisher [19] one knows that on a square lattice of $L \times L$ sites (with periodic boundaries), the specific heat takes its maximum value at temperature $T\left(c_{\max }\right) \simeq T_{c}(1+0.3603 / L)$, where $T_{c} \simeq 2.269185$ is the critical temperature. Combining the results of Refs. [19] and [20] one also has an expansion for the specific heat at the critical temperature:

$$
c\left(T_{c}, L\right)=A_{0} \ln L+C_{0}+\frac{C_{1}}{L}+\frac{C_{2}}{L^{2}}+\frac{C_{3}}{L^{3}}+\cdots
$$

where $A_{0}=(2 / \pi)[\ln (1+\sqrt{2})]^{2} \simeq 0.494358$ and, for an $L \times L$ lattice with periodic boundaries, $C_{0} \simeq 0.138149, C_{1} \simeq-0.170951, C_{2} \simeq 0.018861$, and $C_{3} \simeq 0.056765$. In Fig. 4 we plot $c\left(T_{c}\right)$ versus $\ln L$; a least-squares linear fit yields $c\left(T_{c}\right)=0.498(2) \ln L$, i.e., about $1 \%$ above the exact amplitude. A detailed comparison with theoretical predictions is given in Table I. simulation results agree with theory to within uncertainty. Linear extrapolation of the simulation results for $T\left(c_{\max }\right)$ (for $L \geq 20$ ) versus $1 / L$ yields $T_{c}=2.26966(8)$, that is, about $0.02 \%$ above the exact value of $2.269185 \ldots$ [21]. A similar analysis of the energy per site at $T_{c}$ yields $e_{c}=-1.4147(5)$, consistent with the exact value, $e_{c}=-\sqrt{2}[21]$. As noted above, our sampling method represents the antiferromagnetic (AF) critical point with the same precision as the ferromagnetic one. We have verified that $T\left(c_{\max }\right), c_{\max }$, and $c\left(T_{c}\right)$ associated with the AF transition agree to within uncertainty with the values quoted in 
Table \for the ferromagnetic transition.

FIG. 3: (Color online) Specific heat per site $c$ versus temperature for $L=80$ in five independent simulations.

FIG. 4: (Color online) Specific heat per site at the critical temperature $c_{c}$ versus $\ln L$. The slope of the regression line is $0.498(2)$.

\begin{tabular}{|r|c|c|c|c|}
\hline$L$ & $T\left(c_{\max }\right)(\mathrm{th})$ & $T\left(c_{\max }\right)(\mathrm{sim})$ & $c\left(T_{c}\right)(\mathrm{th})$ & $c\left(T_{c}\right)(\mathrm{sim})$ \\
\hline \hline 10 & 2.35 & $2.34450(6)$ & 1.2600 & $1.2597(3)$ \\
20 & 2.310 & $2.30806(8)$ & 1.6112 & $1.6121(5)$ \\
40 & 2.290 & $2.2889(2)$ & 1.9582 & $1.9573(18)$ \\
80 & 2.2794 & $2.2793(2)$ & 2.3031 & $2.302(3)$ \\
120 & 2.2760 & $2.2761(1)$ & 2.5043 & $2.504(5)$ \\
160 & 2.2743 & $2.2743(2)$ & 2.64695 & $2.655(7)$ \\
\hline
\end{tabular}

TABLE I: Square lattice: comparison of theoretical and simulation results for the temperature of the specific heat maximum, and for the specific heat at $T_{c}$. Note that the theoretical expressions for $T\left(c_{\max }\right)$ and $c\left(T_{c}\right)$ are subject to corrections of order $1 / L^{2}$ and $1 / L^{4}$, respectively.

We turn now to the magnetization, the susceptibility, and Binder's cumulant. The magnetization per site is given by

$$
m(T, L)=\frac{1}{L^{d} Z(T, L)} \sum_{E} \Omega(E, L) e^{-\beta E}\left\langle\left|N_{+}-N_{-}\right|\right\rangle(E, L)
$$

where $\beta=1 / k_{B} T, Z$ is the partition function, and $\left\langle\left|N_{+}-N_{-}\right|\right\rangle(E, L)$ denotes the microcanonical average of the absolute value of the total magnetization $\left(N_{+}\right.$is the number of sites with spin $\sigma=1$ ). Results for the magnetization are plotted in Fig. 5. The uncertainties, which are not visible on the scale of the main graph, are shown in the inset; as is to be expected, they are most severe in the vicinity of the critical point. We note however that in all cases, the relative uncertainty in $m$ is less than $0.4 \%$. The finite size scaling (FSS) relation $m\left(T_{c}, L\right) \sim L^{-\beta / \nu}$ permits one to estimate the associated exponent ratio. Using the data for all system sizes we find $\beta / \nu=0.1237(5)$, that is, $1 \%$ smaller than the exact value of $1 / 8$.

The susceptibility $\chi=\operatorname{var}(M) /\left(L^{d} T\right)$, and its uncertainty are plotted in Fig. 6. The relative uncertainty in $\chi$ is largest (about 1\%) in the critical region, for $L=160$; for smaller 
FIG. 5: (Color online) Magnetization per site versus temperature for system sizes $L=10,20,40$, 80, and 160. Uncertainties are plotted in the inset.

systems and away from the critical region, it is considerably smaller. We list simulation results for the temperature of the susceptibility maximum, $T\left(\chi_{\max }\right)$, and for the susceptibility at $T_{c}$, in Table II. Extrapolating the values of $T\left(\chi_{\max }\right)$ we find $T_{c}=2.26926(10)$. Analysis of the susceptibility data using the FSS relation $\chi\left(T_{c}, L\right) \sim L^{\gamma / \nu}$ yields $\gamma / \nu=1.754(2)$, which is $0.2 \%$ higher than the exact value of $7 / 4$. It is interesting to note that, restricting the analysis to system sizes $20 \leq L \leq 120$, we obtain the slightly superior estimates $\beta / \nu=0.1240(8)$ and $\gamma / \nu=1.748(2)$.

\begin{tabular}{|r|c|c|}
\hline$L$ & $T\left(\chi_{\max }\right)$ & $\chi\left(T_{c}\right)$ \\
\hline \hline 10 & $2.4770(4)$ & $1.7894(4)$ \\
20 & $2.3720(1)$ & $6.093(5)$ \\
40 & $2.3208(2)$ & $20.44(8)$ \\
80 & $2.2949(2)$ & $68.95(25)$ \\
120 & $2.2864(1)$ & $139.5(8)$ \\
160 & $2.28196(14)$ & $233.2(1.8)$ \\
\hline
\end{tabular}

TABLE II: Square lattice: simulation results for the temperature of the susceptibility maximum, and for the susceptibility at $T_{c}$.

FIG. 6: (Color online) Susceptibility per site versus temperature for system sizes $L=10,20,40$, 80, and 160. Uncertainties are plotted in the inset.

An independent estimate of the critical temperature is afforded by Binder's reduced fourth cumulant [23], $Q_{4}=1-\left\langle M^{4}\right\rangle /\left(3\left\langle M^{2}\right\rangle^{2}\right)$. Analysis the crossings of Binder's cumulant for sucessive pairs of system sizes yields the temperatures and cumulant values listed in Table III. Linear extrapolation of the results for the last four pairs yields $T_{c}=2.2694(2)$ and $Q=0.610(1)$. The latter is in good accord with the literature value $Q=0.61071(2)$ [33]. Pooling the estimates for the critical temperature derived from the analysis of the specific heat, the susceptibility, and the reduced cumulant, we find $T_{c}=2.2695(1)$, about $0.01 \%$ above the exact value.

Our estimate for $T_{c}$ is, as noted, based on extrapolations of three sets of size-dependent pseudocritical temperatures, i.e., $T\left(c_{\max }\right), T\left(\chi_{\max }\right)$, and $T_{\times}$. These quantities are plotted 
versus $1 / L$ in Fig. (7. We analyzed the behavior of the pseudocritical temperature associated with the susceptibility maximum, which should follow $T\left(\chi_{\max }\right)-T_{c} \sim L^{-1 / \nu}$. Using our estimate of $T_{c}=2.2695$, a linear fit of $\ln \left[T\left(\chi_{\max }\right)-T_{c}\right]$ versus $\ln L$ yields $\nu=0.99(1)$ consistent with the critical exponent $\nu=1$ for the two-dimensional Ising model.

FIG. 7: (Color online) Two-dimensional Ising model: pseudocritical temperatures (upper to lower) $T\left(\chi_{\max }\right), T\left(c_{\max }\right)$, and $T_{\times}$versus inverse system size. Error bars are smaller than the symbols.

\begin{tabular}{|r|c|c|}
\hline$L, L^{\prime}$ & $T_{\times}$ & $Q_{\times}$ \\
\hline \hline 10,20 & $2.2635(1)$ & $0.61372(10)$ \\
20,40 & $2.2692(2)$ & $0.61115(20)$ \\
40,80 & $2.2689(4)$ & $0.6115(10)$ \\
80,120 & $2.2696(4)$ & $0.6096(17)$ \\
120,160 & $2.2693(5)$ & $0.611(2)$ \\
\hline
\end{tabular}

TABLE III: Ising model, square lattice: simulation results for the temperature and the cumulant value at crossings of Binder's cumulant for pairs of successive system sizes.

As noted above, we find it preferable to perform a relatively small number of long iterations than a large number of shorter ones. To illustrate this point, we performed a series of studies using a total of $5 \times 10^{8}$ LUDS, divided into 5, 10, 25 and 50 iterations, for system size $L=20$. In Fig. 8 we plot the relative uncertainties (estimated using the standard deviation calculated over a set of five independent studies), of $T\left(c_{\max }\right), T\left(\chi_{\max }\right)$, and of the magnetization, susceptibility, and Binder's cumulant, evaluated at the critical temperature. As is clear from the figure, the uncertainties decrease systematically as we reduce the number of iterations; the relative uncertainty is roughly 5-10 times larger using 50 iterations as compared to only five. The inset, for $m_{c}$, illustrates the general trend: despite the monotonic variation of the uncertainties, the estimates obtained using different values of $N$ are mutually consistent.

FIG. 8: (Color online) Relative uncertainties in $T\left(c_{\max }\right)$ (diamonds), $T\left(\chi_{\max }\right)(\times), m_{c}$ (filled squares), $\chi_{c}$ (open squares), and $Q_{c}$ (circles) for $L=20$, versus number of iterations $N$, in studies using a total of $N \times N_{U}=5 \times 10^{7}$ lattice updates. Inset: estimates for $m_{c}$ versus $N$. 


\section{B. Ising model on the simple cubic lattice}

The procedure is essentially the same as for the square lattice. We begin sampling at $L=4$, using the simple initial guess for $s(\rho)$ given in Eq. (6) . Subsequently we study system sizes $L=8,12,16,20,24,28,32$, and 36. The smaller increment in $L$ (as compared with the two-dimensional case), is motivated by faster convergence, and by the need to study a reasonably large number of system sizes in the FSS analysis, which in this case includes a correction to scaling term.

FIG. 9: (Color online) Ising model, simple cubic lattice: specific heat per site versus temperature for system sizes $L=8,12,16,20,24,28,32$, and 36 .

FIG. 10: (Color online) Ising model, simple cubic lattice: pseudocritical temperatures $T\left(c_{\max }\right)$ (lower) and $T\left(\chi_{\max }\right)$ (upper) versus $1 / L^{1 / \nu}$. Inset: temperatures $T_{\times}$of Binder cumulant crossings versus $1 / \bar{L}$ (here $\bar{L}$ denotes the geometric mean of the system sizes).

The specific heat in the critical region is shown in Fig. 9, the relative uncertainty in these results is at most $0.5 \%$. We estimate the critical temperature on the basis of $T\left(c_{\max }\right)$ and $T\left(\chi_{\max }\right)$ (see Fig. 10). Using the values of $T\left(c_{\max }\right)$ for $L=12-36$, a quadratic least-squares fit versus $1 / L^{1 / \nu}$ yields $T_{c}=4.5116(1)$; a similar analysis using $T\left(\chi_{\max }\right)$ yields 4.5114(1). (We use the literature value, $\nu=0.6301(8)$ [24].) These results are in good accord with the best estimate of $T_{c}=4.511528(6)$ [25]. The energy per site at the critical point, $e_{c}(L)$, is essentially linear when plotted versus $1 / L^{1 / \nu}$; extrapolation to infinite size yields $e_{c}=-0.9928(2)$, which compares well with the series-expansion estimate, $e_{c}=-0.9922$ [26]. For each system size, the values of $c_{\max }$ and $T\left(c_{\max }\right)$ associated with the antiferromagnetic critical point are fully consistent with the corresponding ferromagnetic values.

FIG. 11: (Color online) Ising model, simple cubic lattice: magnetization (lower), specific heat (middle), and susceptibility (upper) evaluated at the critical temperature versus system size. Error bars are smaller than symbols.

The critical specific heat, susceptibility, and magnetization are plotted versus system size (on log scales) in Fig. 11. In light of the slight curvature evident in these plots, and given the smaller system sizes used in the three-dimensional studies, we found it useful to include a correction to scaling in the FSS analysis. For example, we fit the data for the magnetization at $T_{c}$ using

$$
\ln m\left(T_{c}, L\right)=-\frac{\beta}{\nu} \ln L+c L^{-y_{i}}+\text { Const. } \equiv f_{L}+\text { Const. }
$$


where $y_{i}$, the dominant irrelevant scaling exponent, is taken as 0.8. (Note that the constant term is not a fitting parameter, as we determine the values of $\beta / \nu$ and $c$ by minimizing the variance between the simulation data and $f_{L}$.) The best-fit parameters for system sizes $L=8$ - 36 are $c=-0.064$ and $\beta / \nu=0.521(12)$. The uncertainty $\sigma_{\bar{\beta}}$ in $\bar{\beta} \equiv \beta / \nu$ is calculated as follows. Let $Y_{L} \equiv \ln m\left(T_{c}, L\right)-f_{L}$, and let $\sigma_{Y}^{2}$ denote the variance of the $Y_{L}$, for parameters $\bar{\beta}$ and $c$ chosen to minimize this variance. Further, let $\sigma_{L}^{2}$ denote the statistical uncertainty in the simulation result for $\ln m\left(T_{c}, L\right)$, and define $\bar{\sigma}_{L}^{2} \equiv \sigma_{L}^{2}+\sigma_{Y}^{2}$. Then we take

$$
\sigma_{\bar{\beta}}^{2}=\sum_{L} \bar{\sigma}_{L}^{2}\left(\frac{\partial \bar{\beta}}{\partial \ln m\left(T_{c}, L\right)}\right)^{2},
$$

where the derivatives are determined numerically. The uncertainties in the other exponent ratios are estimated in a similar manner.

For the susceptibility, we expect the dominant correction to FSS to be $\propto L^{-y_{2}}$ with $y_{2} \simeq 1.96[24]$, and write

$$
\ln \chi\left(T_{c}, L\right)=\frac{\gamma}{\nu} \ln L+c^{\prime} L^{-y_{2}}+\text { Const. }
$$

In this case we find $\gamma / \nu=1.987(4)$. (Using a correction to scaling term $\propto 1 / L$ we instead obtain $\gamma / \nu=1.955(11)$.) A similar analysis of the specific heat data (for system sizes $L=12$ - 36, using a correction term $\propto L^{-\alpha / \nu}$ ) yields $\alpha / \nu=0.161(3)$. Compared with the literature values [25], $\beta / \nu=0.519(2), \gamma / \nu=1.963(3)$, and $\alpha / \nu=0.174(4)$, our results for exhibit errors of $0.4 \%, 1.2 \%$, and $7.5 \%$, respectively. (We note, however, that determination of $\alpha / \nu$ via simulation is in general a difficult task.)

Extrapolation of the crossings of Binder's cumulant (see Fig. 10, inset), yields $T_{c}=$ 4.5124(16), which, while less precise than the estimates cited above, is consistent with the best estimate for the critical temperature. The asymptotic value of Binder's cumulant obtained from the crossings is $Q_{4}=0.47(1)$, while extrapolation of the cumulant values $Q_{4}\left(T_{c}, L\right)$ yields $0.467(1)$. The reference value for the three-dimensional Ising model is $Q_{4}=0.465(3)[24]$.

Entropic sampling is particularly advantageous for data collapse analyses, as it furnishes thermodynamic quantities as continuous functions of temperature. FSS predicts that magnetization curves for diverse system sizes should fall on universal curves (for $T>T_{c}$ and $\left.T<T_{c}\right)$ when plotted in the form $L^{\beta / \nu} m\left(L^{1 / \nu}\left|T-T_{c}\right|\right)$. Figure 12 shows a near-perfect col- 
lapse of the magnetization data for eight system sizes $(L=8-36)$; the associated exponents are $\nu=0.62$ and $\beta / \nu=0.512$. Figure 13 is a similar plot for the susceptibility, again using $\nu=0.62$, and $\gamma / \nu=1.99$. In this case the data for $T>T_{c}$ collapse perfectly while those for $T<T_{c}$ approach a common scaling function with increasing system size. (The downward curvature in these plots signals the low-temperature boundary of the scaling region.) FIG. 12: (Color online) Ising model, simple cubic lattice: scaled magnetization $m^{*} \equiv L^{\beta / \nu} m$ versus scaled temperature $t^{*}=L^{1 / \nu}\left|T-T_{c}\right|$ for eight system sizes $(L=8-36)$, using $\nu=0.62$ and $\beta / \nu=0.512$.

FIG. 13: (Color online) Ising model, simple cubic lattice: scaled susceptibility $\chi^{*} \equiv L^{-\gamma / \nu} \chi$ versus scaled temperature $t^{*}=L^{1 / \nu}\left|T-T_{c}\right|$ for eight system sizes $(L=8-36)$, using $\nu=0.62$ and $\gamma / \nu=1.99$.

\section{Lattice gas with nearest-neighbor exclusion}

We apply our entropic sampling to the lattice gas with nearest neighbor exclusion (NNE) on the square lattice [22, 28, 29], using entropic sampling to estimate the number of distinct configurations $\breve{\Omega}(\mathcal{N}, L)$ with $\mathcal{N}$ particles satisfying the NNE condition, on an $L \times L$ lattice with periodic boundaries. The grand partition function is

$$
\Xi(z, L)=\sum_{\mathcal{N}=0}^{\mathcal{N}_{\max }} z^{N} \breve{\Omega}(\mathcal{N}, L),
$$

where $z=e^{\mu}$ is the fugacity, $\mu \equiv \widehat{\mu} / k_{B} T$ ( $\widehat{\mu}$ denotes the chemical potential), and $\mathcal{N}_{\max }$ is the maximum possible number of particles, equal to $L^{2} / 2$ on the square lattice. (In what follows we refer to $\mu$ as the chemical potential.) The model exhibits an Ising-like phase transition on bipartite lattices; in the ordered phase a majority of the particles occupy one of the sublattices. The associated order parameter is the difference between the occupancies of sublattices A and B:

$$
\phi=\frac{1}{\mathcal{N}_{\max }}\left\langle\left|\sum_{\mathbf{x} \in \mathrm{A}} \sigma_{\mathbf{x}}-\sum_{\mathbf{x} \in \mathrm{B}} \sigma_{\mathbf{x}}\right|\right\rangle,
$$

where $\sigma_{\mathbf{x}}$ is the indicator variable for occupation of site $\mathbf{x}$.

We begin our study with a small system $(L=8)$, using a flat initial distribution, $\ln \Omega_{0}=0$. Following the procedure described in Sec. II, we perform $N=5$ iterations, with increasing numbers of lattice updates [30]. We use ten initial configurations at each iteration: five 
corresponding to an empty lattice, and five in which one sublattice is fully occupied. Here each trial move is either an insertion or a removal of a particle; target sites are chosen at random. For system sizes $L^{\prime}>8$ we construct the initial guess for $\breve{\Omega}\left(\mathcal{N}, L^{\prime}\right)$ using a tenthdegree polynomial fit to $s(\rho, L)=[\ln \breve{\Omega}(\mathcal{N}, L)] / L^{2}$, where $L$ is the previous size studied and $\rho=\mathcal{N} / L^{2}$. We study six sizes in the range $8 \leq L \leq 120$.

Figure 14 shows the susceptibility, $\chi(\mu)=L^{2}\left(\left\langle\phi^{2}\right\rangle_{\mu}-\langle\phi\rangle_{\mu}^{2}\right)$, as a function of the chemical potential for different system sizes; the inset is a similar plot of the order parameter. Estimates for the critical chemical potential $\mu_{c}$ are obtained via analysis of the maxima of the susceptibility and of the compressibility, $\kappa(\mu)=L^{2}\left(\left\langle\rho^{2}\right\rangle_{\mu}-\langle\rho\rangle_{\mu}^{2}\right) /\langle\rho\rangle_{\mu}^{2}$. Extrapolation versus $1 / L$ yields $\mu_{c, \chi}=1.3359(3)$ and $\mu_{c, \kappa}=1.344(2)$. The former, more precise value, is about $0.14 \%$ higher than the literature value. FSS analysis of the susceptibility maxima yields $\gamma / \nu=1.750(2)$, consistent with the exact value for the two-dimensional Ising model.

Using the high-precision result for the critical chemical potential obtained by Guo and Blöte [29], $\mu_{c}=1.33401510027774(1)$, we calculate $\rho_{c}(L), \phi_{c}(L)$ and $Q_{c}(L)$, where $Q=$ $\left\langle\phi^{2}\right\rangle^{2} /\left\langle\phi^{4}\right\rangle$ is related to Binder's reduced cumulant [23]. Linear extrapolation of these quantities versus $1 / L$ yields $\rho_{c}=0.36773(1), \beta / \nu=0.1247(3)$ and $Q_{c}=0.8565(5)$. These results are in very good agreement with the literature values [29, 33]. (Using our own less accurate estimate, $\mu_{c}=1.3359(3)$ we obtain $\beta / \nu=0.122(2)$ and $\rho_{c}=0.36815(3)$.) We summarize our main results for the lattice gas and compare them with reference values in Table IV.

FIG. 14: (Color online) NNE lattice gas: susceptibility versus chemical potential. Inset: order parameter versus chemical potential, system sizes as indicated. Error bars are smaller than the symbols.

\section{Critical singularity of the entropy density}

The critical point corresponds to a value of $\rho$ such that, in the thermodynamic limit, the second derivative $s^{\prime \prime} \equiv d^{2} s / d \rho^{2}=0$. Since direct estimation of the second derivative of numerical data via finite differences is not a viable procedure, we instead use a Gaussian filter, 


\begin{tabular}{llll}
\hline & ES & AWWLS $[22]$ & Literature values \\
\hline$\mu_{c, \chi}$ & $1.3359(3)$ & $1.330(1)$ & $1.33401510027774(1)^{a}$ \\
$\mu_{c, \kappa}$ & $1.344(2)$ & $1.337(2)$ & \\
$Q_{c}$ & $0.8565(5)^{e}$ & $0.852(6)$ & $0.856^{b} ; 0.855(1)^{c} ; 0.85625(5)^{d}$ \\
$\rho_{c}$ & $0.36773(1)^{e}$ & $0.36800(5)^{e}$ & $0.3677429990410(3)^{a}$ \\
$\gamma / \nu$ & $1.750(2)$ & $1.762(8)$ & $7 / 4$ (exact) \\
$\beta / \nu$ & $0.1247(3)^{e}$ & $0.123(2)^{e}$ & $1 / 8$ (exact) \\
\hline
\end{tabular}

${ }^{a}$ Guo and Blöte 29]

${ }^{b}$ Burkhardt and Derrida 31

${ }^{c}$ Nicolaides and Bruce 32$]$

${ }^{d}$ Kamieniarz and Blöte 33 ]

${ }^{e}$ Values obtained using $\mu_{c}$ from 29$]$.

${ }^{f}$ Using our best value for $\mu_{c}$ we obtain $\beta / \nu=0.122(2), \rho_{c}=0.36815(3)$ while the AW study yields $\beta / \nu=0.130(9)$ and $\rho_{c}=0.3675(5)$.

TABLE IV: Critical values for the NNE lattice gas obtained via Entropic Sampling (ES) and WLS with adaptive windows (AW). The results of [32] were obtained via Monte Carlo simulation, those of [29, 31, 33] via transfer-matrix analysis.

$$
s^{\prime \prime}(\rho ; \sigma)=\int_{0}^{2} d \rho^{\prime} s\left(\rho^{\prime}\right) g^{\prime \prime}\left(\rho-\rho^{\prime} ; \sigma\right)
$$

where $g(\rho ; \sigma)$ is a normalized Gaussian distribution with mean zero and standard deviation $\sigma$. (The filter width $\sigma$ is chosen large enough to suppress fluctuations but small enough to maintain resolution; it ranges from about $5 \Delta \rho$ for $L=20$ to about $50 \Delta \rho$ for $L=160$, where $\Delta \rho=1 /\left(2 L^{2}\right)$ is the bond density increment.) Figure 15, for the square lattice, shows that $\left|s^{\prime \prime}\right|$ exhibits a sharp minimum, which appears to approach zero as $L$ increases. The minimum near $\rho=1.7$ corresponds to the ferromagnetic transition; note that the expected (infinite-size) value is $r h o_{c}=1-e_{c} / 2=1+1 / \sqrt{2}=1.707107 \ldots$ The minimum near $\rho=0.3$ corresponds to the antiferromagnetic critical point. Figures 16 and 17 are a similar plots for the Ising model on the simple cubic lattice (for which $\rho_{c}=\left(3-e_{c}\right) / 2 \simeq 1.997$ ), and the NNE lattice gas, respectively.

FIG. 15: (Color online) Ising model, square lattice: $s^{\prime \prime}(\rho)$ versus $\rho$ for system sizes $L=20,40,80$, 120, and 160. The minimum in $\left|s^{\prime \prime}\right|$ grows sharper with increasing system size.

FIG. 16: (Color online) Ising model, simple cubic lattice: $s^{\prime \prime}(\rho)$ versus $\rho=n / L^{3}$ for system sizes $L=16,24$, and 32 . The three curves are indistinguishable at this scale. 
FIG. 17: (Color online) NNE lattice gas, square lattice: $s^{\prime \prime}(\rho)$ versus $\rho=N / L^{2}$ for system sizes $L=20,40,80$, and 120 .

\section{E. A note on restricted sampling}

Our method, as noted, samples the entire range of energies, and while this is advantageous in some circumstances, one might inquire whether the sampling might be restricted or at least concentrated in a region of particular interest, for example, to those energies that occur with a significant probability in the critical region. In Wang-Landau sampling, imposing fixed limits on the sampling range has been found to distort the estimates for $\Omega(E)$ in certain cases [10, 22]. We therefore consider a smooth preference in sampling, in which the acceptance probability, Eq. (2) is modified so:

$$
p_{a}\left(\mathcal{C}^{\prime}\right)=\min \left[\frac{\Omega(E) f(E)}{\Omega\left(E^{\prime}\right) f\left(E^{\prime}\right)}, 1\right] .
$$

Now the probability of visiting energy level $E$ is $\propto 1 / f(E)$, and the formula for updating $\Omega(E)$ becomes,

$$
\Omega_{j+1}(E)=\frac{H_{j}(E)}{\bar{H}_{j}} f(E) \Omega_{j}(E) .
$$

In principle, one can penalize visits to "uninteresting" regions using larger values of $f$, which are then compensated for in the modified updating formula. Since a greater fraction of the sampling occurs in the region of interest, one expects to obtain better statistics there, for the same total number of lattice updates.

We set $f=1$ in the region of interest, that is, for $n \geq n_{\min }$. In one series of studies, $f=\exp \left[\alpha\left(n_{\text {min }}-n\right)\right]$ for $n<n_{\text {min }}$, while in the second we set $f=\exp \left[\alpha\left(n_{\text {min }}-n\right)^{2}\right]$. Here $\alpha$ is a parameter that controls the sharpness of the cutoff in sampling. In the linear case, using $n_{\text {min }} \approx L^{2}$ (i.e., half the maximum bond number), and $\alpha$ in the range $0.1-0.5$, we obtain distributions $\Omega(n)$ (for $L=40$ and 80 ), that differ significantly from those obtained with

uniform sampling, even on the region with $f=1$. In the second set of studies we chose $\alpha$ such that $f(n=0)=e^{-5} \simeq 0.007 ; f$ grows quite slowly in the vicinity of $n_{\text {min }}$. In this case the estimated number of configurations does not exhibit obvious distortions, but we find that the susceptibility and specific heat, evaluated near $T_{c}$, are significantly greater than the 
values obtained with uniform sampling. The relative differences grow with the system size. For example, the maximum susceptibility $\chi_{\max }$ is found to be $0.6 \%(1 \%)$ higher than that obtained using unrestricted sampling, for $L=40$ (80). This occurs despite the fact that at $T_{c}$, the the probability $e^{-\beta E} \Omega(E)$ associated with bond numbers $n \leq n_{\min }$ is $\leq 10^{-30}$, so that the contribution to thermal averages due to this range of $n$ values is completely negligible. We conclude that restricting sampling in this manner does not improve the quality of the results, and in fact appears to cause systematic errors in thermal averages.

\section{SUMMARY}

We have devised an entropic sampling method that covers the entire range of energies, and yields results of good precision for a modest expenditure of computer time. For the two-dimensional Ising model, we obtain the critical temperature to within about $0.01 \%$, the exponent ratios $\gamma / \nu$ and $\beta / \nu$ to within $1 \%$, and excellent agreement with theoretical results for the position and value of the specific heat and susceptibility maxima. Very good results for the Ising model on the simple cubic lattice, and for the lattice gas with nearestneighbor exclusion, are also obtained. The method is relatively simple, and avoids sampling in "windows" with the attendant problems of patching together the results, and of possible distortions at the boundaries.

The sizes used in the present study are relatively modest, and indeed very large sizes are not required to obtain precise results for the Ising model. (One benchmark study of the three-dimensional Ising model [24], for example, uses $L \leq 40$. As the latter work illustrates, precise results for a large set of modest system sizes, coupled with finite-size scaling analysis, may be just as useful as simulations of much larger systems.) In the present study we observe a reduction in quality (as reflected in the relative uncertainty of thermodynamic variables), with increasing system size. We believe that an enriched set of initial configurations, permitting more thorough sampling of configuration space, will help to maintain precision as one increases the system size. This is an important issue for future work.

Key features of our tomographic sampling method are: pooling results starting from very different initial configurations before updating the estimate for $\breve{\Omega}$, and using the results for a given system size to generate an initial estimate for the next larger size. The method 
furnishes globally accurate results, as well as precise estimates for critical properties, using a single set of parameter-free simulations, thereby opening new possibilities for the application of entropic sampling.

\section{Acknowledgments}

We are grateful to CNPq, and Fapemig (Brazil) for financial support.

[1] J. Lee, Phys. Rev. Lett. 71, 211 (1993).

[2] P. M. C. de Oliveira, T. J. P. Penna, and H. J. Herrmann, Braz. J. Phys. 26, 677 (1996).

[3] J.-S. Wang, T. K. Tay and R. H. Swendsen, Phys. Rev. Lett. 82, 476 (1999).

[4] J.-S. Wang and R. H. Swendsen, J. Stat. Phys. 106, 245 (2002).

[5] F. Wang and D. P. Landau, Phys. Rev. Lett. 86, 2050 (2001).

[6] F. Wang and D. P. Landau, Phys. Rev. E 64, 056101 (2001).

[7] B. J. Schulz, K. Binder, and M. Müller, Int. J. Mod. Phys. C 13, 477 (2002).

[8] B. J. Schulz, K. Binder, M. Müller, and D. P. Landau, Phys. Rev. E 67, 067102 (2003).

[9] R. E. Belardinelli and V. D. Pereyra, Phys. Rev. E 75, 046701 (2007); J. Chem. Phys. 127, 184105 (2007); R. E. Belardinelli, S. Manzi, and V. D. Pereyra, Phys. Rev. E 78, 067701 (2008).

[10] A. G. Cunha-Netto, A. A. Caparica, S.-H. Tsai, R. Dickman, and D. P. Landau, Phys. Rev. E 78, 055701(R) (2008).

[11] A. Malakis, A. Peratzakis, and N. G. Fytas, Phys. Rev. E 70066128 (2004).

[12] A. Malakis, S. S. Martinos, I. A. Hadjiagapiou, N. G. Fytas, and P. Kalozoumis, Phys. Rev. E 72066120 (2005).

[13] N. G. Fytas, A. Malakis, and I. Georgiou, J. Stat. Mech. 2008 L07001 (2008).

[14] A. Malakis, A. N. Berker, I. A. Hadjiagapiou, and N. G. Fytas, Phys. Rev. E 79, 011125 (2009).

[15] A. Malakis, A. N. Berker, I. A. Hadjiagapiou, N. G. Fytas, and T. Papakonstantinou, Phys. Rev. E 81041113 (2010). 
[16] By a 'lattice update' we mean one attempted spin flip per site, or $L^{d}$ attempts, using a single-spin-flip algorithm.

[17] M. E. Fisher. Proceedings of the Enrico Fermi International School of Physics, Vol. 51, edited by M.S. Green (Academic Press, Varenna, Italy, 1971). M. E. Fisher and M. N. Barber, Phys. Rev. Lett. 28, 1516 (1972).

[18] M. N. Barber, in Phase Transitions and Critical Phenomena, Vol. 8, edited by C. Domb and J. L. Lebowitz, (Academic Press, New York, 1983).

[19] A. E. Ferdinand and M. E. Fisher, Phys. Rev. 185, 832 (1969).

[20] J. Salas, J. Phys. A 34, 1311 (2001).

[21] H. A. Kramers and G. H. Wannier, Phys. Rev. 60, 252, 263 (1941).

[22] A. G. Cunha-Netto and R. Dickman, Comput. Phys. Comm. 182, 719 (2011).

[23] K. Binder, Z. Phys. B 43, 119 (1981).

[24] H. W. J. Blöte, E. Luijten, and J. R. Heringa, J. Phys. A: Math. Gen. 28, 6289 (1995).

[25] A. L. Talapov and H. W. J. Blöte, J. Phys. A: Math. Gen. 29, 5727 (1996).

[26] M. F. Sykes et al., J. Phys. A: Math. Gen. 5, 640 (1972).

[27] A. M. Ferrenberg and D. P. Landau, Phys. Rev. B 44, 5081 (1991).

[28] L. K. Runnels, Phys. Rev. Lett. 15 (1965) 581.

[29] W. Guo, H. W. J. Blöte, Phys. Rev. E 66 (2002) 046140.

[30] We use $N_{U}=2 n \times 10^{6}$ at the $n$-th iteration.

[31] T. W. Burkhardt and B. Derrida, Phys. Rev. B 32 (1985) 7273.

[32] D. Nicolaides and A. D. Bruce, J. Phys. A: Math. Gen. 21 (1988) 223.

[33] G. Kamieniarz and H. W. J. Blöte, J. Phys. A: Math. Gen. 26 (1993) 201. 